\title{
Nano-Sim: simulating electromagnetic-based nanonetworks in the Network Simulator 3
}

\author{
Giuseppe Piro, Luigi Alfredo Grieco, Gennaro Boggia, Pietro Camarda \\ DEE - Politecnico di Bari, Bari, Italy \\ \{g.piro, a.grieco, g.boggia, camarda\}@poliba.it
}

\begin{abstract}
The progress of nanotechnology is paving the way to the emerging concept of wireless nanosensor network (WNSN). In fact, it is now possible to create integrated machines at the nano scale, which interact on cooperative basis using wireless communications. The research in this field is still in an embryonal stage and the design of the WNSN protocol suite represents a fundamental issue to address. Therefore, an open source simulation framework for WNSN would be highly beneficial to let research activities converge towards participated design methodologies. In an our recent work, we presented a new NS-3 module, namely Nano-Sim, modeling WNSNs based on electromagnetic communications in the Terahertz band. In its preliminary version, Nano-Sim provides a simple network architecture and a protocol suite for such an emerging technology. In this paper, we significantly improved our previous work in several directions. First, we have extended the tool by developing a new routing algorithm and a more efficient MAC protocol. Moreover, focusing the attention on a WNSN operating in a health monitoring scenario, we have investigated how the density of nodes, the transmission range of nanomachines, and the adoption of specific combinations of routing and MAC strategies may affect the network behavior. Finally, a study on Nano-Sim computational requirements has been also carried out, thus demonstrating how the developed module guarantees great achievements in terms of scalability.
\end{abstract}

\section{Categories and Subject Descriptors}

I.6.5 [Simulation and Modeling]: Model DevelopmentModeling methodologies; I.6.7 [Simulation and Modeling]: Simulation Support Systems-Environments

\section{General Terms}

Algorithm, Design, Performance, Verification

Permission to make digital or hard copies of all or part of this work for personal or classroom use is granted without fee provided that copies are not made or distributed for profit or commercial advantage and that copies bear this notice and the full citation on the first page. To copy otherwise, to republish, to post on servers or to redistribute to lists, requires prior specific permission and/or a fee.

WNS3 , Cannes, French Riviera - March 52013

Copyright 2013 ICST, ISBN .

\section{Keywords}

Nanonetworks, wireless nanosensor network, Modeling, Simulation, Performance Evaluation

\section{INTRODUCTION}

In upcoming years, the innovation process triggered by nanotechnologies is expected to foster the development of integrated devices, also known in literature as nanomachines (or nanodevices), with size ranging from one to few hundred of nanometers, very well suited for ICT, biomedical, industrial, and military applications [1]. Due to its limited capabilities, a nanodevice may only execute simple sensing, computing, actuation, and information storage tasks, but this limit can be overcome considering more nanodevices operating in a coordinated fashion and communicating each other [2]. In this way, the overall capability of many of them can be strong enough to create a wireless nanosensor network (WNSN), which can be very useful in several domains.

However, the way information should be exchanged at the nano scale has not yet been defined once for all. Indeed, even if four different communication modes (i.e., nanomechanical, acoustic, molecular, and electromagnetic) have been presented in literature, only those based on molecular diffusion and electromagnetic (EM) radiation seem to be the most suitable for really building a WNSN [4].

The former supposes that nanodevices will be equipped with transceivers able to encode information in molecules, whereas, the latter is based on the transmission and the reception of EM waves.

We remark that the research on nanonetworks is still ongoing and, for the time being, it has been mainly focused on the characterization of the channel at the nano scale. Important contributions are provided in [3] and [10], where sophisticated models for both molecular and EM communications as well as the estimation of the maximum channel capacity have been discussed. Today research, starting from these significant results, as well as considering the general architectural guidelines in [2], is exploring protocol stacks, network architectures, and channel access procedures that could be adopted later on when the technology will be ready to the market.

In this context, a flexible simulation tool would be highly beneficial to support and let research activities converge towards common goals. At the time of this writing, several tools, such as NanoNS [8], N3Sim [13], and the one proposed in [7], have been explicitly conceived for diffusionbased molecular communications. However, we have re- 
cently presented in [16] an open source simulator for EMbased nanonetworks, namely Nano-Sim, developed within the Network Simulator 3 (NS-3) platform ${ }^{1}$. Despite this module poses the basis for the design and the evaluation of more complex network architectures, protocols, and algorithms related to WNSNs, it offers a protocol stack composed by a very simple Media Access Control (MAC), a routing module based on the selective flooding strategy, and a generic unit for generating and processing messages.

In this paper we notably improved our previous contribution in several directions. First of all we extended NanoSim by implementing a more efficient $M A C$ layer and a new random routing algorithm. The selective flooding routing scheme has been ameliorated in order to avoid duplicate forward operations of the same packet, thus preventing an excessive waste of bandwidth. In addition, we carried out a more thorough performance evaluation of a healthmonitoring scenario where nanodevices are diffused into an artery for collecting information about chemical particles and biological functions. In this study, several relevant features have been analyzed to highlight the impact of the density of nodes, the way the transmission range of nanomachines may affect the behavior of the monitoring system, and the performance gain deriving from the adoption of specific combinations of routing and MAC strategies. Finally, a significant scalability study on the computational requirements of Nano-Sim has been also conducted.

The rest of this paper is organized as follows. Sec. 2 describes Nano-Sim as well as summarizes open issues related to WNSNs. The performance evaluation of a health-care system, as well as the scalability test, have been presented in Sec. 3. Finally, Sec. 4 draws the conclusions and discusses planned upgrades of the proposed module.

\section{MODELING EM-BASED NANO NETWORKS}

Nano-Sim has been developed on top of NS-3, an emerging discrete-event and open source network simulator, designed for replacing the popular NS-2 in both research and educational fields. It has been conceived in a modular way to enable graceful upgrades in the future. In addition, its code is freely available under the GPLv2 license to boost its diffusion in the research community [15]. For this reason, we believe that implemented network architecture and protocol stack represent a valid starting point for researchers interested in evaluating novel nanonetworking solutions.

Fig. 1 shows the UML diagram of the most important classes that compose the module, emphasizing the relationship they have with the NS-3 core. It is important to remark that the diagram only reports the most important data members and functions. Some details about relationships among objects have been omitted due to limited space.

The following sub-sections provide a comprehensive presentation of Nano-Sim features, with a major emphasis on the potential contribution it could provide to the study of WNSN open issues.

\subsection{Network Architecture}

The definition of a novel network architecture for WNSNs is crucial to support the communication among nanomachines, as well as their interaction with the external world,

\footnotetext{
${ }^{1}$ NS-3. Network simulator, available at http://www.nsnam . org/.
}

e.g., Internet. As suggested in [2], a generic WNSN should be composed by three kinds of nodes (they may have a fixed position or could be mobile according to the application):

- nanonodes: small and simple devices with very limited energy, computational, and storage capabilities. They can be diffused into a target area for capturing and providing information about the environment.

- nanorouters: nano devices having sizes and resources larger than previous ones. They are in charge of aggregating and processing information coming from nanonodes as well as controlling their behavior by means of short control messages.

- nanointerfaces: most complex nodes able to act as gateways between the nano and the micro scale world, that is they connect the WNSN to the rest of the world. They should be able to convert WNSNs messages to a conventional network system (i.e., WiFi, cellular networks, and so on) and vice-versa.

WNSNs can be adopted several domains, such as biomedical (e.g., health monitoring and drug delivery systems), environmental (e.g., plants monitoring and plagues defeating systems), industrial (e.g., ultrahigh sensitivity touch surfaces and interconnected offices), and military (nuclear, biological and chemical defenses and damage detection systems) [1]. We remark that, according to the application, each of the aforementioned nodes could have a fixed position or could be mobile. For example, in an industrial scenario we can assume the presence of nanomachines in strategic locations for detecting some parameters such as the intensity of the light, the movement of objects and people, the presence of specific chemicals in the air, and so on. In this case, all nodes maintain their position during the Stime, so that the topology of the WNSN is static. On the opposite, in the health-care scenario, we could suppose the presence of nanonodes in a body (i.e., within an artery) able to measure some biological parameters. Despite both nanorouters and nanointerfaces could be placed in particular points of the body, nanonodes should be able to move within the medium (i.e., in our example following the direction of the blood), thus leading to a WNSN with a dynamic topology. For all the envisaged scenarios, the network configuration, the number of nanomachines, the communications among nanonodes, the interactions between them and nanorouters, and the nanointerfaces between a WNSN and the Internet still need to be investigated. Moreover, the design of the entire communication architecture should be carried out taking into account the specific scope of a WNSN: one size fits all solutions cannot be deployed because application domains entailed by such technology have very different requirements.

We modeled within Nano-Sim all the aforementioned kinds of nanomachines, providing, for each of them, basic functionalities that could be improved and customized depending on the scenario to be investigated. Furthermore, the possibility to evaluate both static and mobile scenarios is supportd thanks to the integration of all the mobility models (i.e., constant position, constant acceleration, constant velocity, random walk, random direction, and random waypoints) already available in NS-3.

In Nano-Sima device, which is identified by an unique Dev-ID, has been conceived as a container of several entities, 


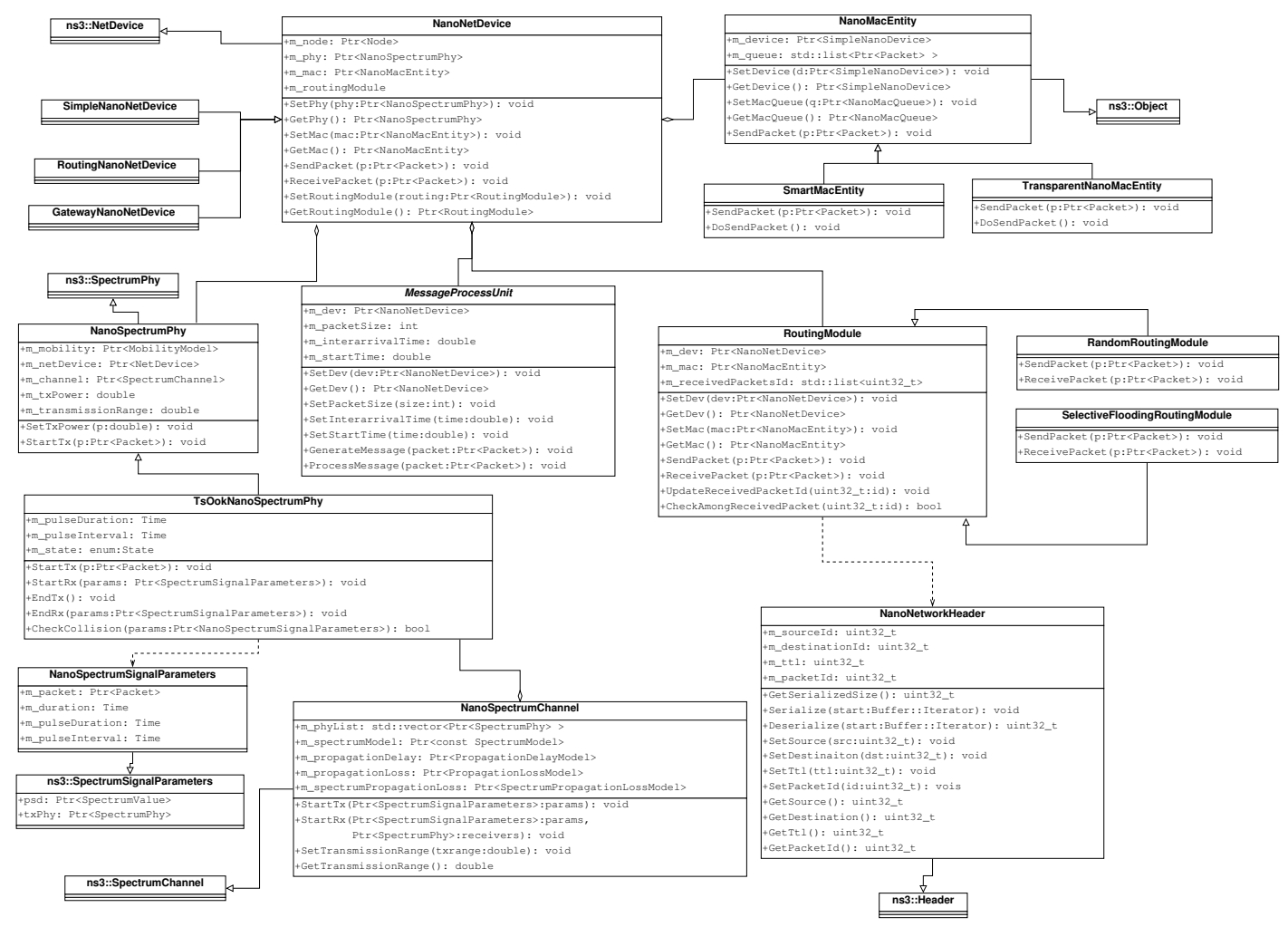

Figure 1: UML class diagram of Nano-Sim.

such as the message processing unit, the network layer, the Media Access Control (MAC) entity, and the PHY interface.

Since the TCP/IP suite cannot be directly adopted in WNSNs [2], we proposed a prototype protocol stack, which is general enough to be considered as a starting point for future optimized solutions.

The task of generating and processing messages is delegated to the Message Process Unit, which can be finely tuned based on the application requirements. The module holds a constant bitrate source that creates periodically fixed-length packets (both interarrival time and packet size can be modified by the user), as it should be the case of WNSNs [2]. Once a new message is created, the Message Process Unit sends it through the protocol stack to the physical (PHY) interface. On the contrary, when a new packet is correctly received from the channel (i.e., no collisions are identified by the PHY interface), it will be delivered to the network layer that will verify if the message has been sent to the node. If so, the application data will be delivered to the Message Process Unit for processing. Otherwise the network layer could decide, according to the routing algorithm, to forward the packet.

\subsection{The Network Layer}

The network layer is in charge of addressing and routing issues. In a WNSN, since nanomachines communicate at the physical layer with very limited transmission ranges, it should be essential to establish a multi-hop path between the sender and the receiver. Hence, the routing algorithm should efficiently handle multi-hop connections. To limit the complexity of routing operations, the WNSN could be organized following a hierarchical architecture [2]. In this way, nanon- odes will be arranged in small clusters, each one having its reference nanorouter, which forwards nanomachine measurements towards the nanointerface. However, We cannot be sure that every nanonode will be able to transmit directly to its closest nanorouter because of its limited transmission range. This means that they should forward packets coming from nanomachines at the edge of the cluster to the reference nanorouter, and viceversa [2].

Nano-Sim provides a flexible network layer that, supporting both selective flooding and random strategies, can be used also to evaluate hierarchical routing operations.

Before delivering the packet to the MAC entity, the network layer, independently on the adopted routing strategy, adds an header composed by five fields: source Dev-ID, sender Dev-ID, next-hop Dev-ID, packet ID, and Time To Live (TTL). Source and sender Dev-IDs identify the device that generated the message and the local node handling the routing strategy, respectively. Meanwhile the former field is set as soon as the message has been received from the Message Process Unit and it remains constant for the entire life of the packet, the latter one is updated hop by hop until the packet reaches the destination node. The next-hop DevID defines the node which the packet will be sent to. The criteria exploited for setting its value depend, as it will be enough discussed below, on the specific routing scheme. The packet ID is assigned by the Message Process Unit, whereas the TTL is set to its default value (i.e., 100) and it is decreased by one unit at each hop ${ }^{2}$. In addition, a flag is

${ }^{2}$ We remark that in a WNSN a message can reach the destination after traversing hundred of nanodevices, this justify why it requires a so high default TTL value. 
added into the network header for indicating if the message should be delivered to the nanointerface or to nanonodes forming the WNSN. Also in this case, its value is provided by the Message Process Unit generating the message. When the network layer receives a packet from the MAC, which is not directed to the receiving node, it will be forwarded again downstream according to the aforediscussed routing strategies.

The selective flooding routing: a node, receiving a message from the upper layer (i.e., by the Message Process Unit it hosts) or from another device through the physical interface, sends the packet to all the devices within its transmission range. In this way, each message generated by a nanonode is suddenly propagated within the WNSN. A similar approach has been already suggested in [4] for molecular-based communications. To prevent an excessive waste of bandwidth, it is very important to avoid duplicate forward operations of the same packet. We remark that each message can be uniquely identified by the pair [packet ID, source Dev-ID]; hence, keeping in memory the pair [packet $I D$, source Dev-ID] associated to latest received messages, a node could not re-transmit packets already received in the past. As default, each nanomachine stores information about at most 20 received packets. In addition, we conceived a further mechanism for reducing the amount of messages sent into the network: with reference to the hierarchical architecture of a WNSN, we imposed that each node must not forward messages in the opposite direction of the destination. In detail, in the case the message is directed to the nanointerface, nanonodes cannot forward the packet if it has been sent by a nanorouter. On the other hand, instead, nanorouters cannot handle a packet received by any nanonodes when the message it contains is directed to latter ones.

The random routing: a nanomachine that has to send a packet selects randomly the next hop among its neighbors. Such an algorithm could be adopted for point-to-point communications, like those between a nanonode and a nanointerface. The random routing assumes that each node knows all the devices within its transmission range. Hence, it must work in couple with a specific MAC strategy able to discover this kind of information; the implemented MAC protocol performing such feature, namely Smart-MAC, will be described below. Whereas the major issue related to the selective flooding approach is to avoid duplicate forward operations, the most important challenge characterizing the random routing algorithm is to prevent the creation of network loops in the multi-hop connection. To this end, each device should avoid to consider as next hop nodes that have already been selected as next hop in the past for a given packet. This task is allowed by keeping in memory the pair [packet ID, next hop Dev-ID] associated to the latest sent messages. Similarly to the previous case, each node stores, as default, information about at most 20 sent packets. To accelerate the delivering of messages to the nanointerface, a nanonode tries to select a nanorouter as the next hop. In fact, it verifies, at the beginning of routing operations, the presence of one or more nanorouters among its neighbors and then it randomly chooses one of them, subjected to the constraints imposed by the previously described loop avoidance scheme. Otherwise, if no nanorouters in the neighborhood are identified, it has to send the packet to a nanonode. From another hand, since we consider only communications between nanonodes and nanointerface, we imposed that a nanorouter cannot choose a nanonode as the next hop. As a final consideration, we remark that a given device could not be able to select a next hop because it has not any neighbors or nodes within its transmission range cannot be considered according to the constraints imposed by the routing algorithm itself. In this case, the packet will be enqueued at the MAC layer and the routing protocol will delegate the MAC entity to select the next hop in the future (see the description of the Smart-MAC strategy).

\subsection{Channel access procedures}

For all wireless technologies, the design of a channel access procedure is a very difficult task. In a WNSN, it becomes even more critical due to the very high number of nodes expected in such systems. In this context, protocols requiring the synchronization among nodes are, in general, not recommended [2]. In addition, considering that the most suitable transmission techniques are based on pulse-based communications (more details will be provided in next sub-section), approaches based on carries sensing strategies, like the Carrier Sense Multiple Access (CSMA), cannot be applied due to the lack of a signal to sense. Therefore, asynchronous MAC algorithms are the best candidates for a WNSN: they allow packet transmission without any channel contention. Moreover, thanks to their very low computational requirements, asynchronous MAC schemes are also easy to implement on resource constrained nanodevices.

In line with these premises, we implemented at the MAC layer two different asynchronous strategies: Transparent$M A C$ and Smart-MAC. We decided to not include further headers at the MAC layer: all information useful for delivering messages to the destination node are included within the header added at the network layer.

Transparent-MAC: it is a very simple solution, which let packets be transmitted from the network layer to the physical interface without executing any kind of control.

Smart-MAC: it represents a more efficient algorithm. A packet received from the upper layer is not immediately delivered to the physical interface, but it is stored into a dedicated queue. Before transmitting it, the MAC exploits a handshake procedure for discovering nanomachines within its transmission range. If at least one node is found, the packet is sent to the PHY layer. Moreover, if the random routing algorithm is enabled and the next hop has not been yet selected (see the previous sub-section for details), the MAC layer chooses it randomly among nanomachines within its transmission range. In the case the node has not any neighbors, the MAC entity applies a random backoff delay prior to starting again the handshake procedure. It is worth to note that this delay is uniformly extracted in the range [minimum backoff time, maximum backoff time], whose boundaries can be modified by the user. Finally, in the presence of multiple enqueued packets, each new transmission is scheduled after a specific time interval, which is computed through the same backoff mechanism. This additional technique has been introduced for reducing the probability to have physical collisions.

\subsection{Channel and Physical models}

Nanoantennas may support EM communications in the Terahethz band (i.e., $0.1 \div 10.0 \mathrm{THz}$ ). The entire spectrum can range from a few hundred of gigahertz to almost $10 \mathrm{THz}$, 
thus enabling a channel capacity in the order of few terabit/s and a transmission range that cannot exceed few tens of millimeters. [10]. Due to the size and energy constraints of nanomachines, classical communication techniques based on the transmission of signals with long duration, which are typically adopted in wireless sensor network (WSN) [14], cannot be used in a WNSN. Considering the huge capacity of the Terahertz channel, a valid solution is to exchange very short pulses (i.e., each one lasting some femto or pico seconds) spread over the entire spectrum. The most promising modulation scheme allowed for the nano technology, is the Time Spread On-Off Keying (TS-OOK) [11]. With TSOOK, a logical 1 is transmitted by using a short pulse and a logical 0 is encoded as a silence. It is important to observe that this scheme is able to ensure, at the sender side, both high energy and communication efficiency. For what concern the receiver, instead, it just should be equipped by an energy detection system in order to notice the presence or the absence of a pulse. Due to technological limitations (i.e., the communication unit can work only with a very low duty-cycle), the only drawback affecting the TS-OOK modulation scheme is that the time between two consecutive pulses should be much longer than the pulse duration. On the other hand, it offers two important advantages. Firstly, it does not require that nanodevices should be synchronized before starting the transmission of the packet. Moreover, it causes the sharing of the medium among multiple users. In fact, since the time between the transmission of two consecutive pulses has to be much longer than the pulse duration, several nanodevices can concurrently send sequence of pulses which are slightly time-shifted, without incurring in collisions.

TS-OOK parameters (such as the duration, the transmission frequency, and the energy of pulses) need to be carefully tuned according to the application scenario and to the upper layers of the protocol stack. To this aim, Nano-Sim provides a PHY layer based on TS-OOK and allows its parameters to be easily adjusted based on the user needs.

The characterization of the channel at the nano scale has been discussed in [10] and [9]. These works demonstrated that the maximum channel capacity depends on the medium composition, the distance among nodes, the power transmission capability of a nanomachines, and TS-OOK parameters. As a consequence, a WNSN must be accurately designed taking into account all properties of the scenario of interest and of the target behavior that have to be reached for the considered system.

At the time of this writing, Nano-Sim models the impact of the channel behavior by means of the the knowledge of the physical transmission range (note that the same approach is frequently adopted by other famous simulators like NS-2 and Omnet++). During the simulation, due to the limited transmission range that we expect at the Terahertz band, nanomachines will be split in different broadcast domains. For this reason, the channel can deliver a packet to a given node (thus triggering the reception procedure at the PHY layer) if its distance with respect to the source does not exceed the transmission range threshold. An user can exploit sophisticated channel models, like those presented in [10] and [9], for properly setting the transmission range of nanomachines and some physical parameters. Then, focusing the attention on a specific application field of its interest, he can test performances of the WNSN and the protocol stack devised by itself from a system level point of view.

Anyway, in order to favour future upgrades of the developed module, we have implemented channel and physical entities by extending libraries provided within the Spectrum Framework [5]. With the Spectrum Framework, in fact, it will be possible to implement more complex models that may model the selectivity of the Teraherz channel in both frequency and time domains.

According to the TS-OOK modulation scheme, the message is sent by means of a sequence of pulses. We remark that simulating the transmission and the reception of each single pulse would unnecessarily increase the complexity of Nano-Sim, due to the very high number of nodes belonging to a WNSN. For this reason, the packet transmission is handled at the system level as depicted in the following:

1. the MAC entity, after the channel access procedure, calls the method of the PHY interface which is in charge of packet transmission.

2. The PHY layer creates the data structure associated to the signal to transmit, storing details such as, the time instant when the transmission starts, the pulse duration, $T_{p}$, the pulse transmission interval , $T_{i}$, and the transmission duration, txTime. In particular, the total transmission duration, is computed as

$$
\text { txTime }=\left[(L-1) \cdot T_{i}\right]+T_{p}
$$

where $L$ represents the packet length expressed in bits.

3. The aforementioned data structure is delivered to the channel and then sent to all the nodes which are in the transmission range of the sender.

4. The destination node handles the reception procedure. In particular, it verifies if there are physical collisions during the time interval required for receiving the packet, i.e., txTime. A collision occurs if pulses belonging to different transmissions overlap in the time domain. In order to detect this episode, a nanomachines stores transmission parameters (i.e., those listed at the point 2 ) associated to all active reception procedures. Once a reception procedure ends, the node exploits these parameters for re-building the sequence of received pulses during the time interval required for receiving the considered packet. In this way, it is able to evaluate the presence of overlapped pulses. If the packet is correctly received, it will be forwarded to upper layers of the protocol stack. Otherwise, all collided messages will be deleted.

\section{PERFORMANCE EVALUATION}

The aim of this section is twofold: first, it is intended for demonstrating the practical utility of the developed tool in the biomedical field, by studying a health-monitoring system based on WNSN. Then, it investigates the simulator scalability in terms of computational requirements (i.e., simulation time and memory usage) in order to understand NanoSim limits and forecasts future research.

\subsection{Analysis of a Health-monitoring System}

The WNSN we considered is composed by a number of nanonodes, nanorouters, and a nanointerface deployed in 


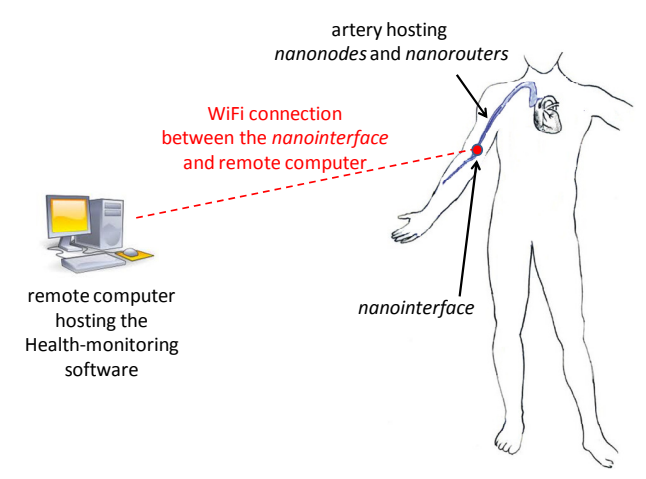

Figure 2: The studied health-monitoring system.

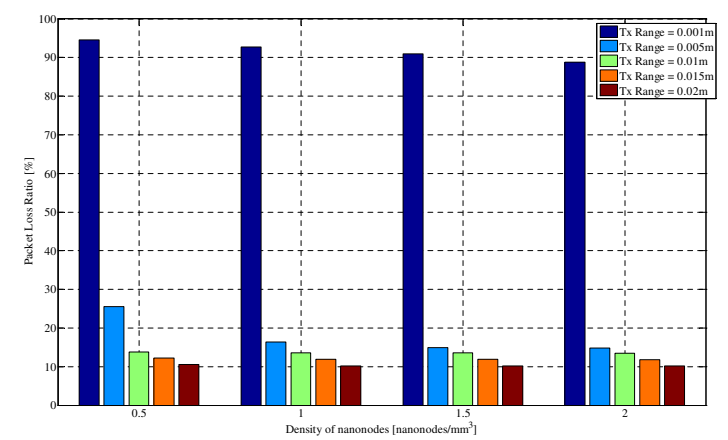

(a)

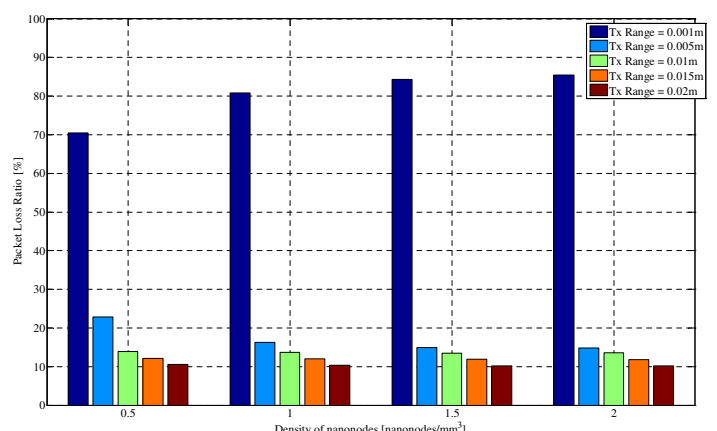

(b)

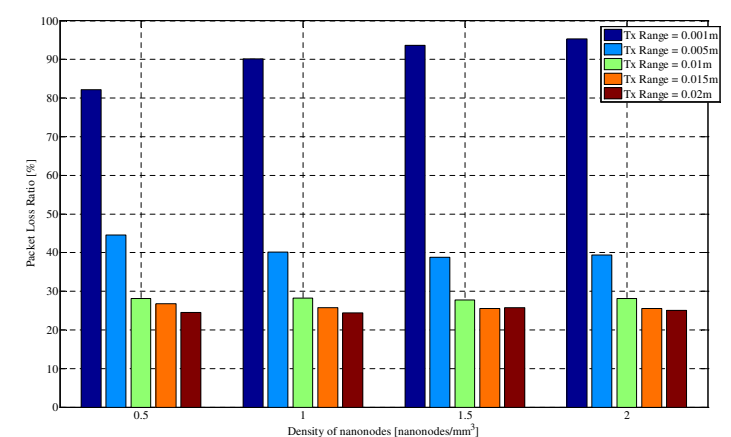

(c)

Figure 3: PLR achieved in WNSNs with (a) selective flooding and Transparent-MAC, (b) selective flooding and Smart-MAC, and (c) random routing and Smart-MAC protocols.

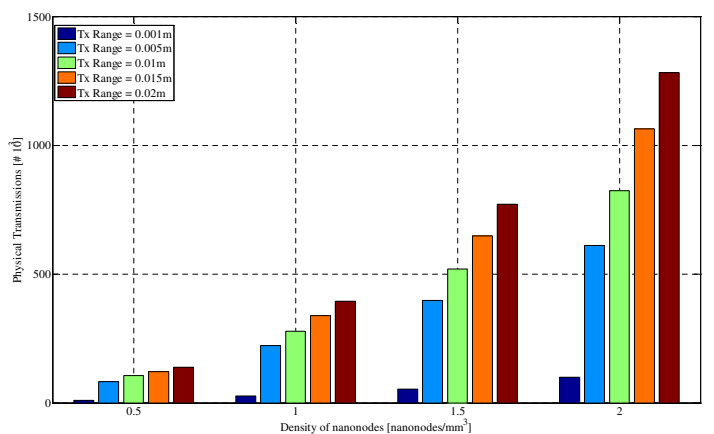

(a)

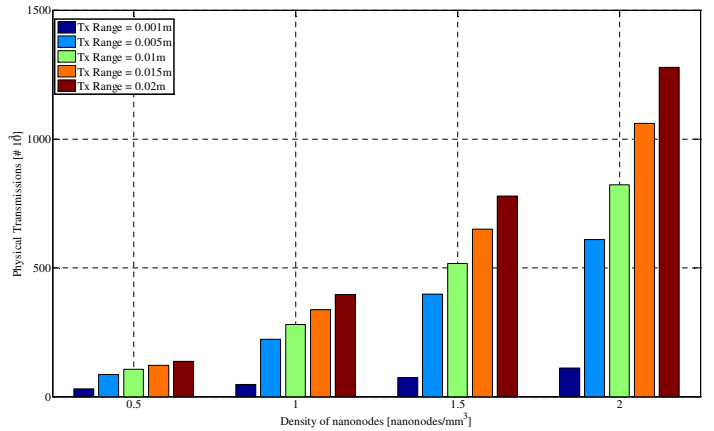

(b)

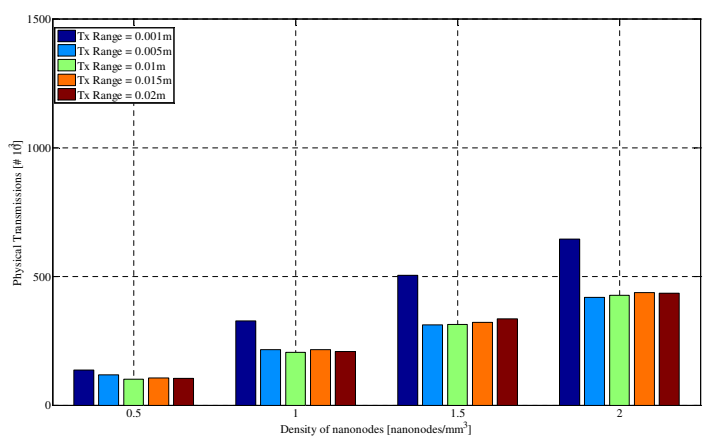

(c)

Figure 4: Number of physical transmission handled in WNSNs with (a) selective flooding and Transparent-MAC, (b) selective flooding and Smart$M A C$, and (c) random routing and Smart-MAC protocols.

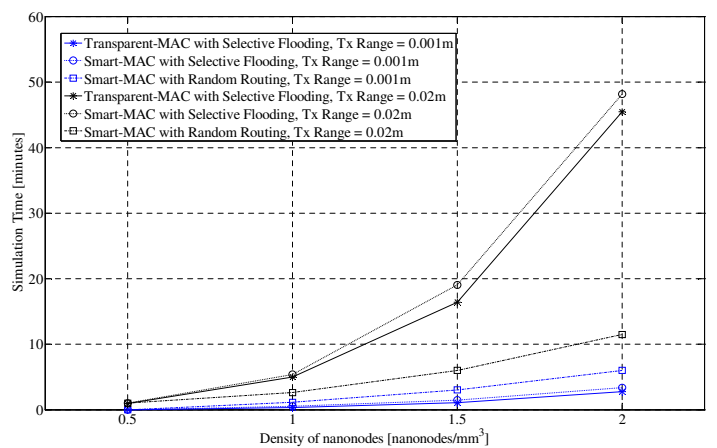

Figure 5: Simulation time vs number of nanonodes. 
Table 1: Simulation Parameters.

\begin{tabular}{l|l} 
Parameter & Value \\
\hline \hline System parameters & \\
Simulation duration & $5 \mathrm{~s}$ \\
Density of nanonodes & {$[0.5-2]$ nodes $/ \mathrm{mm}^{3}$} \\
Number of nanointerfaces & 1 \\
$\begin{array}{l}\text { Number of nanorouters } \\
\text { Artery size }\end{array}$ & 50 \\
\hline \hline PHY details & $10^{-3} \times 10^{-3} \times 1.15 \mathrm{~m}^{3}$ \\
$\begin{array}{l}\text { Pulse energy } \\
\text { Pulse duration }\end{array}$ & $100 \mathrm{pJ}$ \\
Pulse Interarrival Time & $100 \mathrm{fs}$ \\
TX range of nanonodes & $10 \mathrm{ps}$ \\
TX range of nanorouters & {$[0.001-0.02] \mathrm{m}$} \\
\hline MAC & $0.02 \mathrm{~m}$ \\
Backoff interval (only for the & {$[0 \mathrm{~ns}, 100 \mathrm{~ns}]$} \\
Smart-MAC) & \\
\hline Network Layer & 100 \\
Initial TTL value & $128 \mathrm{bytes}$ \\
\hline MessageProcessingUnit & $0.1 \mathrm{~s}$ \\
\hline $\begin{array}{l}\text { Packet size } \\
\text { Message generation time interval }\end{array}$ &
\end{tabular}

an artery of the arm, whose length and diameter have been set to $30 \mathrm{~cm}$ and $1 \mathrm{~mm}$, respectively [6].

At the beginning of the simulation, nanonodes are uniformly distributed within the first $15 \mathrm{~cm}$ of the artery. Then, they move along the artery following the direction of the blood at the speed of $20 \mathrm{~cm} / \mathrm{s}$ [6]. Nanorouters are uniformly distributed inside the entire artery, whereas the nanointerface is deployed at the center of it. Both of them maintain their position fixed during the time.

All nanonodes are equipped with a sensing unit and are able to sense the surrounding environment and to collect information about chemical particles and biological functions. Starting from these measurements, they generate, periodically, messages that are sent, through multi-hop paths, to the closest nanointerface, which is connected to the health remote server by means of a IEEE 802.11 wireless connection (see Fig. 2).

Focusing the attention on the aforedescribed scenario, we studied the impact of the density of nanomachines and their physical transmission range on the behavior of the healthcare system. We tested three different protocol stacks composed by the following mix of routing and MAC algorithms: (i) selective flooding routing protocol with Transparent-MAC, (ii) selective flooding routing protocol with Smart-MAC, and (iii) random routing protocol with Smart-MAC. We note that the random routing cannot be adopted together with the Transparent-MAC strategy because, as highlighted in the previous section, it must work in couple with a MAC protocol able to discover nodes within the transmission range of the sender.

Simulation settings are summarized in Tab. 1. We remark that the PHY layer has been configured according to parameters suggested in [12]. In line with the study presented in [10], according to which the transmission range of nanomachines cannot exceed few tens of millimeters, we evaluated the network behavior for different values of trans- mission ranges, selected in the range $[0.001,0.02] \mathrm{m}$.

Fig. 3 shows the percentage of packets that are not received by the nanointerface, i.e., the Packet Loss Ratio (PLR), measured for all the considered scenarios. The first interesting result is that the PLR decreases as the density of nanonodes and their transmission range increase because there are more chances to find a multi-hop path to the nanorouter. This finding is relevant and should be very carefully accounted for sizing a WNSN. In details, the transmission range equal to $0.001 \mathrm{~m}$ entails, independently from adopted routing and MAC protocols, as well as from the number of nodes, the worst results: the PLR is so high that it becomes unacceptable. This underlines that a WNSN cannot guarantee the delivery of messages between nanonodes and the remote server, and viceversa, when the composition of the medium (i.e., the blood in the considered example) and the characteristics of the physical interface allow very limited transmission capabilities. When the transmission range is equal to $0.005 \mathrm{~m}$, the WNSN is able to deliver a higher amount of messages to the remote server. Moreover, a smaller and smaller PLR could be registered by increasing the node density. For higher transmission ranges, instead, the PLR is lower thanks to the capability of the WNSN to correctly establishing the multi-hop connection between the node generating the message and the nanointerface.

We can observe that, the adoption of the random routing algorithm leads to a slight increase of the percentage of lost messages. In fact, the random selection of the next hop may prevent some packets to reach the destination before the end of the simulation or before the expiration of the TTL. When the selective flooding strategy is adopted at the network layer, the Smart-MAC procedure is able to guarantee better performance (expressed in terms of a lower PLR) only in scenarios where it is very difficult to establish the multihop connection (e.g., see results achieved with the transmission range equal to $0.001 \mathrm{~m}$ ). In this case, all additional mechanisms implemented with the considered MAC protocol could increase the probability to send messages to nodes that, being within the transmission range of the sender, are able to receive them correctly. However, no evident performance gains can be observed for other network conditions.

Hence, we can conclude saying that the Transparent-MAC could be the best MAC scheme to use in couple with the selective flooding routing because it requires few computational capabilities. For the random routing, instead, the Smart-MAC is the only possible solution for the reasons already discussed in Sec. 2.

The number of physical transmissions handled in the WNSN has been pictured in Fig. 4. When the selective routing algorithm is used, physical transmissions increase with the density of nanonodes because there are more devices that generate messages to sent to the remote server. Moreover, the highest the transmission range, the highest the number of physical transmissions. This result can be justified considering that all nodes receiving a packet are allowed to perform forwarding operations and that the amount of these devices increases by enhancing the transmission range. In the case the transmission range of nanosensors is set to $0.001 \mathrm{~m}$, the random routing algorithm registers the highest number of physical transmissions because the random selection of the next hop leads to longer multi-path connections. A different behavior is achieved for higher transmission range values: in these conditions the number of physical transmissions is 
fewer than the one measured for the selective flooding algorithm. The reason is that for each sent packet only one node in the network (i.e., the next hop selected by the routing algorithm) can perform forwarding operations.

To summarize, the presented analysis demonstrates that the PLR is a critical issue in a WNSN. We found that the random routing is able to guarantee a good compromise between the registered PLR and the number of physical transmissions. Anyway, with the aim of further improving network performances, sophisticated MAC and routing strategies as well as a careful tuning of the network size are required. In general, an optimized trade-off among protocol complexity and network size is a relevant topic in WNSNs that Nano-Sim will help to solve in the future.

\subsection{Scalability Test}

To provide a further insight, we evaluate the scalability of the proposed module in terms of both simulation time and memory usage on a Linux machine with a $2.6 \mathrm{GHz} \mathrm{CPU}$ and 4 GBytes of RAM. We considered a WNSN with settings equal to those used before, focusing the attention to a sub set of transmission ranges. We have measured that the memory usage is always less that $250 \mathrm{MB}$ and no significantly differences have been obtained when increasing the number of nodes. On the other hand, we observed that the simulation time (see Fig. 5) increases with the transmission range of nanomachines because there are more nodes able to receive and forward messages in a WNSN. Furthermore, among all considered protocol suites, the one exploiting the random routing at the network layer requires the lowest simulation time because of a limited number of physical transmission handled in the network. Finally, all results registered for the conducted tests represent, without any doubts, great achievements in terms of scalability.

\section{CONCLUSION AND FUTURE WORKS}

This paper presents some enhancements we added to NanoSim, i.e., an open source tool modeling WNSNs within the NS-3 simulator. Moreover, a deeply analysis on theoretical assumptions considered during the development of the module and a more complete performance evaluation of a WNSN operating in a health-monitoring system have been also provided. Thanks to its extremely modularity, NanoSim has all the characteristics to become a reference tool for researchers working in the area of nanonetworks. As next steps of our work, we plan to extend the simulator by implementing new features, e.g., more sophisticated routing, MAC, and PHY protocols and models. Furthermore, more complex applications in both medical and industrial fields will be also investigated. Finally, we will try to merge the module into the official release of NS-3.

\section{Acknowledgment}

This work was supported by the PON projects (ERMES-0103113, DSS-01-02499, EURO6-01-02238) funded by the Italian MIUR, and by the Apulia Region Project PS 025 (ICT supporting logistic services: a model of organized market).

\section{REFERENCES}

[1] I. Akyildiz and J. Jornet. Electromagnetic wireless nanosensor networks. Nano Communication Networks, 1(1):3-19, Mar. 2010.
[2] I. Akyildiz and J. Jornet. The internet of nano-things. IEEE Wireless Communications, 17(6):58 -63, Dec. 2010.

[3] I. F. Akyildiz, J. MiquelJornet, and M. Pierobon. Propagation models for nanocommunication networks. In Proc. of the Fourth European Antennas and Propagation Conference, EuCAP, pages $1-5$, Apr. 2010.

[4] B. Atakan and O. B. Akan. Carbon nanotube-based nanoscale ad hoc networks. IEEE Communications Magazine, 48(6):129 -135, Jun. 2010.

[5] N. Baldo and M. Miozzo. Spectrum-aware channel and phy layer modeling for ns3. In Proc. of ICST VALUETOOLS, Pisa, Italy, October 2009.

[6] D. G. Cramp and E. R. Carson. The Circulatory System. Taylor \& Francis, 1986.

[7] L. Felicetti, M. Femminella, and G. Reali. A simulation tool for nanoscale biological networks. Nano Communication Networks, Oct. 2011.

[8] E. Gul, B. Atakan, and O. Akan. Nanons: a nanoscale network simulator framework for molecular communications. Nano Communication Networks, 1(2):138-156, Oct. 2011.

[9] J. Jornet and I. Akyildiz. Channel Capacity of Electromagnetic Nanonetworks in the Terahertz Band. In Proc. of IEEE Int. Conf. on Commun., ICC, pages $1-6$, May 2010.

[10] J. Jornet and I. Akyildiz. Channel modeling and capacity analysis for electromagnetic wireless nanonetworks in the terahertz band. IEEE Transactions on Wireless Communications, 10(10):3211 -3221, Oct. 2011.

[11] J. Jornet and I. Akyildiz. Information capacity of pulse-based wireless nanosensor networks. In Proc. of IEEE Conf. on Sensor, Mesh and Ad Hoc Communications and Networks, SECON, pages 80 -88 , Jun. 2011.

[12] J. M. Jornet, J. C. Pujol, and J. S. Pareta. PHLAME: A Physical Layer Aware MAC protocol for Electromagnetic nanonetworks in the Terahertz Band. Nano Communication Networks, 3(1):74 - 81, 2012.

[13] I. Llatser, I. Pascual, N. Garralda, A. Cabellos-Aparicio, and E. Alarcon. N3sim: a simulation framework for diffusion-based molecular communication. IEEE Technical Committee on Simulation, 8:3-4, 2011.

[14] M. R. Palattella, N. Accettura, X. Vilajosana, T. Watteyne, L. A. Grieco, G. Boggia, and M. Dohler. Standardized protocol stack for the internet of (important) things. IEEE Commun. Survs. Tut., 2013. to be published.

[15] G. Piro. Nano-sim - The open source framework for simulating EM-based WNSNs. [OnLine] Available: http://telematics.poliba.it/nano-sim.

[16] G. Piro, L. A. Grieco, G. Boggia, and P. Camarda. Simulating wireless nano sensor networks in the ns-3 platform. In in Proc. of Workshop on Performance Analysis and Enhancement of Wireless Networks, PAEWN, Barcelona, Spain, Mar. 2013. 